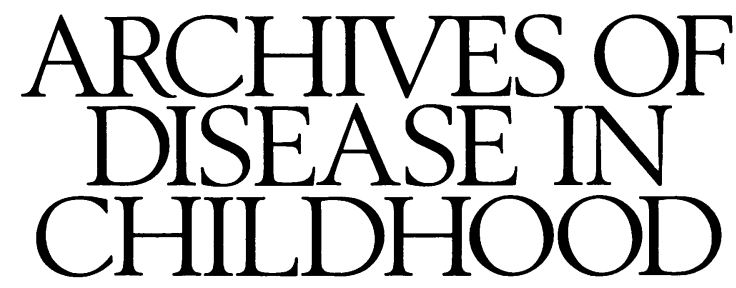

The fournal of the British Paediatric Association

\title{
Annotation
}

\section{Antibiotic treatment of suspected neonatal meningitis}

Neonatal meningitis remains a life threatening emergency with a mortality of $20-50 \% ; 50 \%$ of the survivors sustain major sequelae. ${ }^{12}$ Fortunately the condition is uncommon, the incidence being about $0 \cdot 4$ to 1 per thousand live births. ${ }^{3}$ Early diagnosis and treatment are vital if these figures are to improve. The commonest organisms are still group B streptococcus and Escherichia coli, although the range is wide. ${ }^{4}$ Listeria monocytogenes is now the third most common cause in the United Kingdom. Staphylococcus epidermidis is an important pathogen after invasive neurosurgical procedures.

\section{Choice of antibiotic}

Meningitis is more often suspected than confirmed because the signs are usually subtle and non-specific. Lumbar punctures are therefore often performed as part of a septic screen. If the infant is very ill at the time and probably bacteraemic there is a risk of seeding organisms into the meninges. In this situtation treatment should include an antibiotic that is known to penetrate the cerebrospinal fluid. Our current recommendation is cefotaxime $(100 \mathrm{mg} / \mathrm{kg} / 24$ hours divided into two doses), penicillin ( $100 \mathrm{mg} / \mathrm{kg} / 24$ hours divided into two doses), and gentamicin $(2.5 \mathrm{mg} / \mathrm{kg}$ every 12 hours or every 18 hours for infants less than $1000 \mathrm{~g}$ with adjustment according to drug concentrations) with early rationalisation of treatment once the results of microscopy of the cerebrospinal fluid and culture are known. Listeria will not respond to cephalosporins, therefore cover with this agent alone will be inadequate. Chloramphenicol ( $50 \mathrm{mg} / \mathrm{kg} / 24$ hours in two divided doses) is now not a good choice ${ }^{56}$; the results are unimpressive, the therapeutic margin is narrow, and organisms readily become resistant. Furthermore, the drug is not bactericidal against the anaerobic Gram negative bacilli and can interfere with the bactericidal effect of gentamicin. ${ }^{7}$ It is subject to dosage and dilution errors and cannot be dialysed out rapidly by the peritoneal route if an overdose has been given. Chloramphenicol is metabolised to its inactive metabolites by microsomal glucuronyl transferase. This enzyme system is immature in the newborn, and is the route for metabolism of other drugs including the anticonvulsants phenobarbitone and phenytoin, which are often required in babies with meningitis. If chloramphenicol is used access to a rapid drug assay is needed and this is rarely available.

\section{$E$ coli and other Gram negative organisms}

These still present major problems despite the advent of the cephalosporins. Although the cumulative experience with these agents in the neonate must be greater than the 26/240 patients reported in 12 trials, ${ }^{8}$ mortality has not improved as much as one would have hoped. Cefotaxime is currently the most effective cephalosporin; there have been treatment failures with cefuroxime, which is not always effective against Gram negative organisms, and ceftazidime should be reserved for pseudomonas infections. Gentamicin should be continued primarily to treat the associated septicaemia.

Cerebral ultrasound should always be performed as soon as possible. If the ventricles are enlarged with a ventricular index measurement above the 97 th centile ${ }^{9}$ there is no doubt in our minds that intraventricular treatment should be used. Intrathecal treatment using the lumbar route of administration is ineffective, probably due to a failure of drug to reach the ventricular cerebrospinal fluid. Intraventricular treatment should be administered by a surgically implanted Rickham reservoir with daily instillation of $1 \mathrm{mg}$ of gentamicin for seven days. The system allows measurement and control of cerebrospinal. fluid pressure, together with bacteriological monitoring and avoids the problems associated with the development of porencephalic cysts along the needle tracks of repeated ventricular punctures. In a trial involving 61 centres in the United States and South America, McCracken and his colleagues reported the outcome of 52 infants randomised to receive systemic treatment plus intraventricular treatment compared with systemic treatment alone. ${ }^{10}$ The study was stopped early because of a higher mortality in the group given intraventricular treatment, but many of the infants had salmonella infection and were treated relatively late. There has been no study of intraventricular treatment using treatment by a reservoir with concomitant systemic administration of cephalosporins and little information on the ventricular drug concentration achieved after systemic treatment alone in dilated ventricles.

When the ventricles are normal in size we would do a diagnostic ventricular tap under ultrasound control if there was no clinical improvement at between 24 and 48 hours, taking the opportunity to instil a single dose of gentamicin and to measure the cerebrospinal fluid pressure. If the examination of the fluid confirmed that ventriculitis was present we would ask our neurosurgical colleagues to 
attempt to insert a Rickham reservoir in order to administer intraventricular antibiotics. In babies who were improving at $24-48$ hours we would repeat the lumbar puncture and only perform a ventricular tap if organisms were still seen.

It is generally accepted that treatment should be continued for three weeks after sterilisation of the cerebrospinal fluid. Progress should be monitored by following the platelet count, white cell count, and $\mathrm{C}$ reactive protein concentration; there should be repeat lumbar puncture if any of these are failing to follow the expected improvement. Ventricular size should be monitored by ultrasound three times a week. A computed tomogram is occasionally necessary to diagnose subdural collections or abscess formation. Proteus has a particular prediliction for abscess formation, ${ }^{11}$ therefore all patients in whom this organism is grown should have a computed tomogram.

The outlook for Gram negative meningitis remains grim, with a $20 \%$ mortality and with $38 \%$ of the survivors suffering major sequelae. We feel that an improvement can only be made if the suggested aggressive approach is adopted.

\section{Organisms other than coliforms}

Group B streptococcus still accounts for most cases of neonatal meningitis. The outlook is better than that for Gram negative infections and treatment should be for three weeks with penicillin and gentamicin. Dual treatment is advised because of the synergy between these two agents. Intraventricular treatment is seldom, if ever, necessary.

$L$ monocytogenes is an emerging cause of meningitis in babies. Both ampicillin and penicillin are extremely effective in high dose, and either of these should be combined with gentamicin. The prognosis is better than for Gram negative meningitis.

A special problem arises in infants with ventriculoperitoneal shunts. These are prone to infection, with many units reporting a $30 \%$ infection rate after first insertion despite perioperative antistaphylococcal prophylaxis with flucloxacillin and vancomycin. $S$ epidermidis is the usual culprit and the system needs to be exteriorised, after which both systemic antibiotics and intraventricular vancomycin treatment should be used. Caution is required before reinsertion of the system, ideally only when the cerebrospinal fluid has been sterile for at least two weeks.

\section{Conclusion}

Antibiotics alone do not treat meningitis, and the importance of intensive care cannot be overstressed. Most babies have an associated bacteraemia and many are shocked. Circulatory support using massive colloid/blood transfusion and inotropes, aggressive anticonvulsant treatment necessitating ventilation and the possible need for neurosurgical intervention mean that infants with Gram negative meningitis should be transferred to a unit with appropriate facilities and expertise. Those infected with other organisms may be cared for in units without intensive care facilities. Over the next decade there may be more information about the use of immunoglobulin and granulocyte transfusions in severe neonatal infections.

G GANDY, J RENNIE

Rosie Maternity Hospital,

Robinson Way,

Cambridge $C B 22 S W$

1 Davies PA. Bacterial and fungal infections. In: Levene MI, Bennett MJ, Punt J, eds. Fetal and neonatal neurology and neurosurgery. New York. Churchill Livingstone, 1988:427-44.

2 Bell AH, Brown D, Halliday HL, McClure G, McReid M. Meningitis in the newborn - a 14 year review. Arch Dis Child 1989;14:873-4

3 Overall JC. Neonatal bacterial meningitis. $\mathcal{f}$ Pediatr 1970;76:499-511.

4 De Louvois J, Blackbourn J, Hurley J, Harvey DR. Meningitis during the first year of life - a 2 year prospective study. Arch Dis Child 1988;63:698.

5 Bell WE, McGuiness GA. Suppurative CNS infection in the neonate. Semin Perinatol 1982;6:1-25.

6 Levin M. Treatment of meningitis. Pediatric Reviews and Communications 1989;2:3-8.

7 Rahal J, Simberkoff MS. Bactericidal and bacteriostatic action of chloramphenicol against meningeal pathogens. Antimicrob Agents Chemother 1979; phenicol

8 Feldstein TJ, Uden DL, Larson TA. Cefotaxime for treatment of gramnegative meningitis in infants and children. Pediatr Infect Dis 1987;6:471-4.
. Levene MI. Measurement of the growth of lateral ventricles in preterm infants with real time ultrasound. Arch Dis Child 1981;56:900-4.

10 McCracken GH Jr, Mize SG, Threkheld N. Intraventricular gentamicin therapy in gram-negative bacillary meningitis of infancy: report of the second neonatal meningitis study group. Lancet 1980;i:787-91.

11 Renier D, Flandin C, Hirsch E, Hirsch J-F. Brain abscesses in neonates. A study of 30 cases. $\mathscr{f}$ Neurosurg 1988;69:877-82. 